\title{
Sun-like Stars: magnetic fields, cycles and exoplanets
}

\author{
Rim Fares \\ INAF - Osservatorio Astrofisico di Catania, Via Santa Sofia 78, 95123 Catania, Italy \\ email: rfares@oact.inaf.it
}

\begin{abstract}
In Sun-like stars, magnetic fields are generated in the outer convective layers. They shape the stellar environment, from the photosphere to planetary orbits. Studying the largescale magnetic field of those stars enlightens our understanding of the field properties and gives us observational constraints for field generation dynamo models. It also sheds light on how "normal" the Sun is among Sun-like stars. In this contribution, I will review the field properties of Sun-like stars, focusing on solar twins and planet hosting stars. I will discuss the observed large-scale magnetic cycles, compare them to stellar activity cycles, and link that to what we know about the Sun. I will also discuss the effect of large-scale stellar fields on exoplanets, exoplanetary emissions (e.g. radio), and habitability.
\end{abstract}

Keywords. stars: magnetic fields, stars: activity, technique: polarimetry

\section{Introduction}

More than a century after the discovery of the magnetic nature of sunspots (Hale 1908), and after achieving milestones in our understanding of solar magnetism, many questions remain unanswered. The Sun, despite having high spatial and temporal resolution, is nonetheless a single case study. Investigating the magnetism of Sun-like stars offers us the opportunity to place the Sun among its analogs, in order to have a wider view. The knowledge we have from solar studies regarding small and large-scale fields, as well as solar cycles, can guide, on the other hand, our exploration of magnetism in stars.

The solar magnetic field evolves in time. The latitude of emergence of sunspots, those small-scale magnetic features of thousands of Gauss, migrate from middle values toward the equator over a timescale of 11 years. This gives the butterfly diagram, showing a cyclic behaviour that I will refer to as the activity cycle. The large-scale field, which has a mean value that is much weaker (few Gauss), switches polarity every 11 years, giving a cycle length of about 22 years (see, e.g. Solanki et al.(2000) and Mordvinov et al.(2012) for a discussion about radial and azimuthal fields' evolution). During solar minimum, the large-scale field has a simple dipolar configuration, that becomes more complex during the solar maximum.

Stellar activity cycles are observed by studying activity proxies such as Calcium H\&K and X-rays, see e.g. Baliunas et al.(1995), Metcalfe et al.(2010), Sanz-Forcada et al.(2013) and Berdyugina(2005). Solar-like stars have their magnetic fields generated by dynamo mechanisms in their outer convective layers. Studying their magnetism can thus give insights and observational constraints on dynamo generation theories. In this review, I will present the results of large-scale magnetic field studies of solar-like stars with masses between 0.7-1.5 $\mathrm{M}_{\odot}$. The results presented here are obtained through the MagIcS initiative (a Magnetic Investigation of various classes of Stars), and in particular from the 
Star-Planet Interaction (http://lamwws.oamp.fr/exo/starplanetinteractions/ SPIScience) and the Bcool (http://bcool.ast.obs-mip.fr/) threads of this consortium.

\section{Methodology: mapping of the magnetic field}

Magnetic fields, when present in stellar photospheres, can cause Zeeman splitting and polarisation of spectral lines. Zeeman broadening is hard to detect in sun-like stars at optical wavelength (Anderson et al. 2010). The polarisation, on the other hand, can be detected. Its properties depend on the relative position of the observer to the orientation of the magnetic field. Circular polarisation, for example, is sensitive to the line-of-sight component of the field (see Landi Degl'Innocenti \& Landolfi 2004).

To map stellar magnetic fields, one can use tomographic imaging technique, called Zeeman-Doppler Imaging (ZDI). This technique relies on the fact that when the star rotates, we observe different parts of the stellar disc. If the magnetic field distribution is not homogeneous on the stellar surface, for each rotational phase, a different polarisation signature can be observed. ZDI consists of inverting circular polarisation profiles into the magnetic topology (strength, distribution, polarity) of the field that produces such signatures (Semel 1989). The reconstruction is an ill-posed problem, a regularisation technique should be used to reconstruct a unique map (Maximum entropy, e.g. Brown et al.(1991) and Hussain et al.(2000); Tikhonov regularisation, e.g. Piskunov \& Kochukhov(2002)). Results shown in this review are obtained using a maximum entropy regularisation, and describing the field by its poloidal and toroidal components, all described using spherical harmonics expansions (Donati et al. 2006a). We note however that ZDI does not recover small-scale field, since the spatial resolution is limited, and the signatures of small-scale fields can cancel out in some field geometries.

Polarisation spectra can be collected using spectropolarimeters, such as ESPaDOnS at CFHT, its twin instrument NARVAL at TBL, or HARPSpol at La Silla (Donati et al. 2006b, Aurière 2003, Piskunov et al. 2011). The signal in these spectra is extremely small, usually within the noise level. In order to improve the Signal-to-Noice (S/N), a multiline technique dubbed Least-Square Deconvolution (LSD, Donati et al.(1997), Kochukhov et al. (2010))is used. It improves the $\mathrm{S} / \mathrm{N}$ by a factor $\sim 30$ relative to single lines.

\section{Magnetic properties and cycles of Sun-like stars}

I will review, in this section, the current picture we have about the cool-stars' magnetism. But before that, I will discuss the magnetic properties of two stars with different stellar properties.

HD 76151 has been investigated in the Bcool program. It is a G3 star, with a mass of $1.24 \pm 0.12 M_{\odot}$ and a $v \sin i$ of $1.2 \pm 0.5 \mathrm{~km} \mathrm{~s}^{-1}$. It was observed in the beginning of 2007 . Fig. 1 show its reconstructed magnetic map. Radial, azimuthal and meridional magnetic field are reconstructed. The mean magnetic energy is of 5.6 \pm 2 Gauss (Petit et al. 2008), the field is mainly a simple dipolar radial field. HD 189733, on the other hand, is a cooler active star (K2) that hosts a transiting hot Jupiter. It was observed under the SPI program. It has a mass of $0.82 \pm 0.03 M_{\odot}$ and a $v \sin i$ of $2.97 \pm 0.22 \mathrm{~km} \mathrm{~s}^{-1}$ (Moutou et al. 2007). The reconstructed map for July 2008 (Fares et al. 2010) shows a mean magnetic field of 36 Gauss, with a dominant azimuthal toroidal component as shown in fig.1.

These two examples show that the magnetic field of different solar-like stars can have different strengths and topologies. All the stars mentioned in this review are solar-like, 

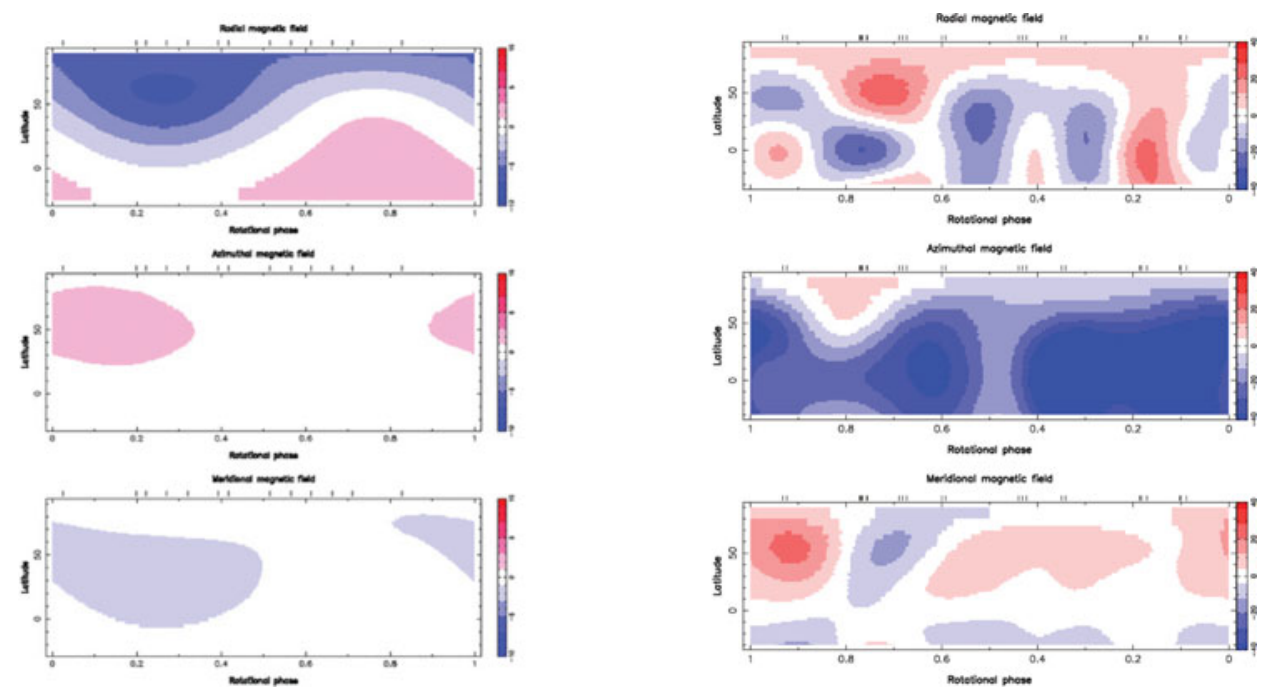

Figure 1. Left panel: The three components of the field in spherical coordinates system are presented for HD 76151 (from Petit et al. 2008). Right panel: The magnetic map of HD 189733, adapted from Fares et al.(2010). Please not that the colour scale is not the same for the left and the right maps.

with an outer convective envelope. We compare their properties in a mass-rotation diagram, since both the mass and the rotation are important in this context. The strength of the field, the percentage of poloidal energy and how axisymmetric the poloidal field is are considered, because they are important ingredients for dynamo. Data were compiled from Moutou et al. (2007); Donati et al. (2003, 2008); Petit et al. (2008, 2009); Jeffers \& Donati(2008); Donati \& Landstreet(2009); Fares et al.(2009,2010,2012,2013); Morgenthaler et al.(2011,2012) and Marsden et al.(2011). Fig. 2 shows the magnetic properties in the mass-rotation plane. As mentioned before, different properties appear in this diagram. However, they follow a trend that is different if the star's Rossby number is greater or lower than one. Stars with Rossby $>1$, including the Sun, have smaller, dominantly axisymmetric poloidal field. The Sun, in this perspective, is not different from its analogs.

This is the general picture we have currently for magnetism in those cool stars. But the question one can ask is: what about magnetic cycles?

As mentioned in the introduction, stars exhibits activity cycles. A spectropolarimetric follow-up has been done for some targets. The first star to show a large-scale magnetic cycle with polarity switches was $\tau$ Boo, a massive planet hosting F-star (Donati et al.(2008), Fares et al.(2009), Fares et al.(2013)). The rotation of the star is synchronised with the planet. The magnetic field of $\tau$ Boo has a mean value of about 5 Gauss. A polarity flip was observed between June 2006 and June 2007, and another flip was observed between June 2007 and June-July 2008, indicating a fast cycle of 2 years (or less). The field was mainly poloidal for all these epochs. Observed in January 2008 between two plarity flips, the field showed an important toroidal component, indicating a change in configuration between the flips. It was first suggested that the planet might have played a role synchronising the outer convective layers of the star, and thus affecting the dynamo. But other stars (without hot Jupiters) have shown fast polarity reversals, indicating that fast cycles exist in cool Stars (e.g. F star (HD78366), and G dwarf (HD190771)). Note that for some of these stars, unlike the solar case, reported activity cycles are longer than the observed magnetic cycles. Most of the stars with polarity flips are fast rotators, 

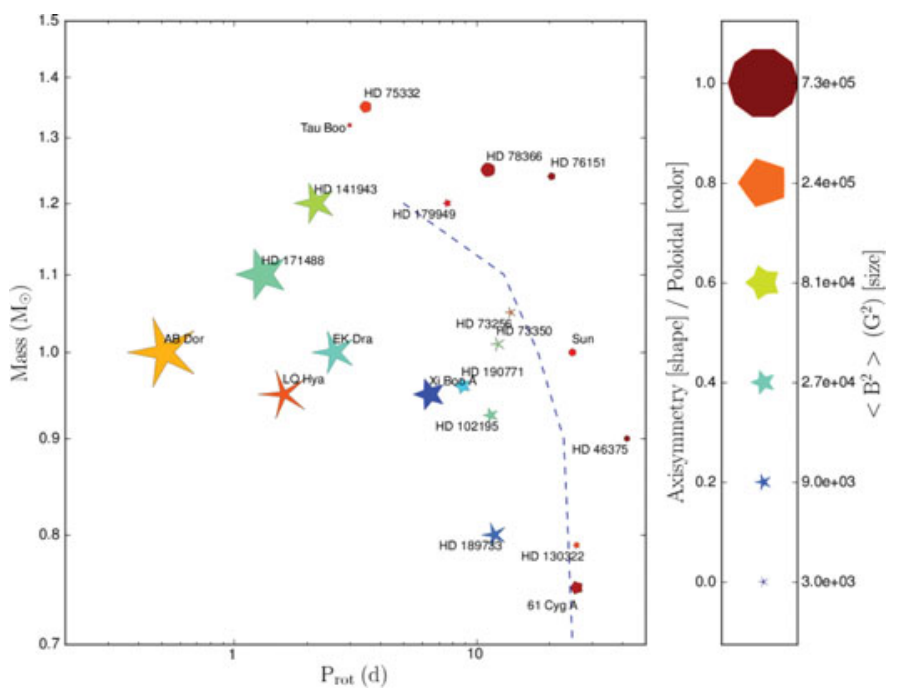

Figure 2. Mass-rotation diagram of reconstructed magnetic fields of Sun-like stars. The dashed line shows Rossby number of unity. The size of the symbol represents the field strength, its color the contribution of the poloidal component to the field, and its shape the degree axisymmetry of the poloidal component.

however, a polarity flip is observed on the slow-rotator 61 Cyg (Boro Saikia et al. in prep). This new result indicates that it is possible to detect magnetic cycles on slow rotators, now that we have long time-span of observations.

\section{Exoplanet's environment}

Stellar active regions can mimic the signal of an exoplanet. In order to detect earth-like planets, stellar activity should be well understood and modelled. Characterising exoplanets, however, is not limited to determining their masses, radii, and orbits. It also consists of studying their environment, their emissions (e.g. radio) and their interactions with their surrounding (i.e. stellar wind). It is possible to model more realistically the stellar corona when the large-scale magnetic field is reconstructed based on spectropolarimetric observations. The magnetic map can be used as a boundary condition for modelling the corona. This allows us to better understand the magnetic environment around the planet. Such environment can be responsible for bow shock formation (Vidotto et al. 2011) and radio emission (e.g., Zarka 2007, Jardine \& Collier Cameron 2008). If the bow shock or the radio emission is observed simultaneously with spectropolarimetric observations of the star, one can indirectly infer the planetary magnetic field. A planetary magnetic field can act as a shield protecting the planet from the stellar wind. Earth-mass planets around M dwarfs, the focus of future missions, are subject to "aggressive" environment because of the intense stellar fields. Modelling the corona and the planetary orbit's environment is needed to understand better the conditions of habitability around active stars.

\section{Conclusions}

In this review, I presented the technique and results used to study the large-scale magnetic fields of solar-like stars. Spectropolarimetry, the study of polarisation in the spectral lines, and Zeeman-Doppler Imaging allow the reconstruction of the large-scale field maps. These techniques delivered, in the last 25 years, great knowledge on stellar magnetism. Magnetic fields of cool stars have different properties related to the strength of the field, the percentage of the energy in the poloidal to toroidal components, and how axisymmetric the field is. Stars with Rossby number greater than one seem to have similar properties while stars with a Rossby lower than one have different properties when 
compared to the first group, while they share between them similar properties. Magnetic cycles have been detected in Sun-like stars, mostly around fast rotators. Different types of cycles have been found, regular and complex cycles. The magnetic field polarity flips every year for at least one of the stars observed, much faster than the solar case. The length of the activity cycle to the large-scale cycle can be different from the solar case (11 vs 22 years for the Sun). Being able to access stellar activity data to review activity cycle length in the shadow of large-scale cycle finding is an important step to understand better stellar magnetism. Collecting more data to study activity, whether in the calcium $\mathrm{H} \& \mathrm{~K}$, other activity tracers, X-rays (corona) is important to understand directly the link between small-scale, large-scale, and even larger-scale (corona) activity cycles. This will help understand field dynamo generation and improve our theories.

\section{References}

Anderson, R. I., Reiners, A., \& Solanki, S. K. 2010, A\&A, 522, A81

Aurière, M. 2003, EAS Publications Series, 9, 105

Baliunas, S. L., Donahue, R. A., Soon, W. H., et al. 1995, ApJ, 438, 269

Berdyugina, S. V. 2005, Living Reviews in Solar Physics, 2, 8

Brown, S. F., Donati, J.-F., Rees, D. E., \& Semel, M. 1991, A $\& A$, 250, 463

oro-Saikia et al., in preparation

Donati, J.-F., Semel, M., Carter, B. D., Rees, D. E., \& Collier Cameron, A. 1997, MNRAS , 291, 658

Donati, J.-F., Collier Cameron, A., Semel, M., et al. 2003, MNRAS, 345, 1145

Donati, J.-F., Howarth, I. D., Jardine, M. M., et al. 2006a, MNRAS, 370, 629

Donati, J.-F., Catala, C., Landstreet, J. D., \& Petit, P. 2006b, Astronomical Society of the Pacific Conference Series, 358, 362

Donati, J.-F., Moutou, C., Farès, R., et al. 2008, MNRAS, 385, 1179

Donati, J.-F. \& Landstreet, J. D. 2009, ARAA, 47, 333

Fares, R., Donati, J.-F., Moutou, C., et al. 2009, MNRAS, 398, 1383

Fares, R., Donati, J.-F., Moutou, C., et al. 2010, MNRAS, 406, 409

Fares, R., Donati, J.-F., Moutou, C., et al. 2012, MNRAS, 423, 1006

Fares, R., Moutou, C., Donati, J.-F., et al. 2013, MNRAS, 2010

Hale, G. E. $1908, A p J, 28,315$

Hussain, G. A. J., Donati, J.-F., Collier Cameron, A., \& Barnes, J. R. 2000, MNRAS, 318, 961

Jardine, M. \& Collier Cameron, A. 2008, A\&SA, 490, 843

Jeffers, S. V. \& Donati, J.-F. 2008, MNRAS, 390, 635

Kochukhov, O., Makaganiuk, V., \& Piskunov, N. 2010, A\&̋A, 524, A5

Landi Degl'Innocenti, E. \& Landolfi, M. 2004, Astrophysics and Space Science Library, 307

Marsden, S. C., Jardine, M. M., Ramírez Vélez, J. C., et al. 2011, MNRAS, 413, 1922

Metcalfe, T. S., Basu, S., Henry, T. J., et al. 2010, ApJ(Letters), 723, L213

Mordvinov, A. V., Grigoryev, V. M., \& Peshcherov, V. S. 2012, Solar Physics, 280, 379

Morgenthaler, A., Petit, P., Morin, J., et al. 2011, AN, 332, 866

Morgenthaler, A., Petit, P., Saar, S., et al. 2012, ApJ, 540, A138

Moutou, C., Donati, J.-F., Savalle, R., et al. 2007, A\&AA, 473, 651

Petit, P., Dintrans, B., Solanki, S. K., et al. 2008, MNRAS, 388, 80

Petit, P., Dintrans, B., Morgenthaler, A., et al. 2009, ApJ, 508, L9

Piskunov, N. \& Kochukhov, O. 2002, A\&A, 381, 736

Piskunov, N., Snik, F., Dolgopolov, A., et al. 2011, The Messenger, 143, 7

Sanz-Forcada, J., Stelzer, B., \& Metcalfe, T. S. 2013, A\&AA, 553, L6

Semel, M. 1989, A\&A, 225, 456

Solanki, S. K., Schüssler, M., \& Fligge, M. 2000, Nature, 408, 445

Vidotto, A. A., Jardine, M., \& Helling, C. 2011, MNRAS, 414, 1573

Zarka, P. 2007, P\&SS, 55, 598 